\title{
A Model-Driven Approach for Conducting Simulation Experiments
}

This paper was downloaded from TechRxiv (https://www.techrxiv.org).

\section{LICENSE}

CC BY 4.0

SUBMISSION DATE / POSTED DATE

20-10-2021 / 29-10-2021

\section{CITATION}

Wilsdorf, Pia; Heller, Jakob; Budde, Kai; Zimmermann, Julius; Warnke, Tom; Haubelt, Christian; et al. (2021): A Model-Driven Approach for Conducting Simulation Experiments. TechRxiv. Preprint. https://doi.org/10.36227/techrxiv.16836868.v1

$\mathrm{DOI}$

10.36227/techrxiv.16836868.v1 


\title{
A Model-Driven Approach for Conducting Simulation Experiments
}

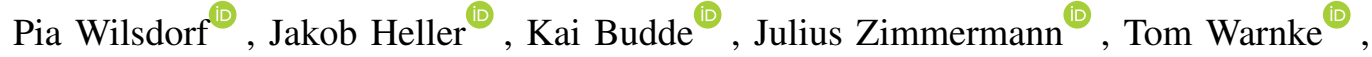 \\ Christian Haubelt $^{\circledR}$, Dirk Timmermann ${ }^{(0)}$, Ursula van Rienen ${ }^{\circledR}$, Member, IEEE, \\ and Adelinde M. Uhrmacher
}

\begin{abstract}
With the increasing complexity of simulation studies, and thus increasing complexity of simulation experiments, there is a high demand for better support for their conduction. Recently, model-driven approaches have been explored for facilitating the specification, execution, and reproducibility of simulation experiments. However, a more general approach that is suited for a variety of modeling and simulation areas, experiment types, and tools, which also allows for further automation, is still missing. Therefore, we present a novel model-driven engineering (MDE) framework for simulation studies that extends the state-of-theart by means for knowledge sharing across domains, increased productivity and quality of complex simulation experiments, as well as reusability and automation. We demonstrate the practicality of our approach using case studies from three different fields of simulation (stochastic discrete-event simulation of a cell signaling pathway, virtual prototyping of a neurostimulator, and finite element analysis of electric fields), and various experiment types (global sensitivity analysis, time course analysis, and convergence testing). The proposed framework can be the starting point for further automation of simulation experiments, and therefore can assist in conducting simulation studies in a more systematic and effective manner. For example, based on this MDE framework, approaches for automatically selecting and parametrizing experimentation methods, or for planning following activities depending on the context of the simulation study, could be developed.
\end{abstract}

Index Terms-Computer simulation, knowledge representation, formal specifications, software reusability, model-driven development, design for experiments

\section{INTRODUCTION}

$\mathbf{M}$ ODELING and simulation has become a key tool in many sciences and engineering disciplines [1]. A simulation study is usually characterized by iterative model refinements, intertwined with problem analysis, conceptual modeling, and various experimentation activities, as illustrated in modeling and simulation life cycles [2]-[4] (see Fig. 1). Due

Manuscript received ??? ??, ???; revised ???? ??, ???

P. Wilsdorf, K. Budde, T. Warnke, and A. M. Uhrmacher are with the Institute for Visual and Analytic Computing, University of Rostock, 18051 Germany.

J. Heller, C. Haubelt, and D. Timmermann are with the Institute of Applied Microelectronics and Computer Engineering, University of Rostock, 18051 Germany.

J. Zimmermann, and U. van Rienen are with the Institute of General Electrical Engineering, University of Rostock, 18051 Germany.

E-mail: firstname.lastname@uni-rostock.de .

This work was funded by the DFG (German Research Foundation) Collaborative Research Center 1270/1 - 299150580 ELAINE and the DFG research project UH 66/18 GrEASE. to its complexity, there is a high demand for approaches that allow simulation studies to be conducted in a more effective and systematic manner. Especially support for conducting simulation experiments is of increasing interest, as they drive important activities, such as the calibration, validation, and analysis of models and are essential for the reproducibility of simulation results. With new computational power, new availability of data, and the strengthened role of simulation experiments in advancing science within the different domains, the requirements referring to simulation studies and experiments become more rigorous, the variety and complexity of simulation experiments increases, and the field of modeling and simulation is rapidly evolving.

To support simulation experiments, a clear separation of concerns between model and experiment is necessary [5]. Following the reproducibility crisis [6], a variety of approaches have been developed for capturing simulation experiments explicitly and machine-accessibly. This is reflected, for example, in domain-specific languages such as SESSL ("Simulation Experiment Specification via a Scala Layer") [7], [8] and SED-ML ("Simulation Experiment Description Markup Language"), or reporting guidelines such as MIASE ("Minimum Information About a Simulation Experiment") [9] and the reporting guidelines for finite element analysis studies in biomechanics [10]. Also modeling and simulation frameworks, such as the Simulation Automation Framework for Experiments (SAFE) [11], plug-in based approaches [12], or approaches for packaging simulation experiments as reproducible objects [13] add to the reproducibility and portability of simulation experiments.

Model-driven engineering (MDE) based approaches can provide additional support in the specification and execution of simulation experiments. In an MDE approach, meta models require certain experiment inputs, guide users through the experiment specification, and finally generate executable code [14]. Current MDE approaches for simulation experiments realize and demonstrate this for the generation of experiment designs for parameter scans [14] and hypothesis testing [15]. However, they concentrate on specific fields of simulation, support only a specific experiment type, or are tightly coupled with specific tools. As a consequence, their applicability and thus their impact on the way simulation experiments are conducted is limited. Also, some typical 
benefits of MDE [16], such as improved knowledge sharing and further automation, are not yet available or not fully exploited for simulation experiments. Thus, a more general approach is desirable that can support simulation experiments more broadly and thus assist in conducting entire simulation studies more effectively and systematically.

In this paper, we present a novel MDE framework that enhances the state-of-the-art of conducting simulation experiments in the following ways:

- Improved knowledge sharing across domains: Experiment meta models make knowledge about the structure and ingredients of simulation experiments explicit. By building various kinds of meta models and storing them in repositories, we allow modelers and developers to share knowledge about simulation experiments between the different communities, e.g., finite element analysis or virtual prototyping. These usually develop their simulation methodology and tooling independently, although the way simulation experiments are specified and conducted rarely differ and thus could be supported by the same pipeline.

- Increasing productivity and quality for complex experiments: For novice modelers, MDE clearly facilitates the specification of simulation experiments via targeted graphical user interfaces and code generation. We provide additional support via an experiment validator and allow for support of complex simulation experiments via meta model composition. Due to the focus on complex experiments, also experienced modelers can benefit.

- Reusability: The meta models give meaning to the ingredients of simulation experiments. Together with an easyto-use, tool-independent format and a variety of backend bindings, we support the flexible and automatic reuse of simulation experiments. This is of interest when the performance of different simulation tools needs to be compared [17], or model alternatives implemented using different modeling and simulation approaches shall be evaluated [18].

- Automation: Further automation of simulation experiments can be achieved if the knowledge about the various simulation experiments (given by meta models) is put into relation with context knowledge about the simulation study (e.g., given by conceptual models [19], provenance [20], or documentation [21]), as in these cases, simulation experiment specifications may be automatically generated and executed [22]. As our approach provides means for integration with various other frameworks, it presents a valuable basis for further automation of simulation experiments.

We demonstrate the above features and benefits of the approach using concrete examples from three case studies. The studies are part of the Collaborative Research Centre (CRC) 1270 ELAINE $^{1}$, which aims to develop novel electrically active implants for bone and cartilage regeneration as well as for deep brain stimulation. In each of the three studies a different modeling and simulation approach/domain is in focus (i.e., stochastic discrete-event simulation of a cell signaling

\footnotetext{
${ }^{1}$ https://www.elaine.uni-rostock.de/en/
}

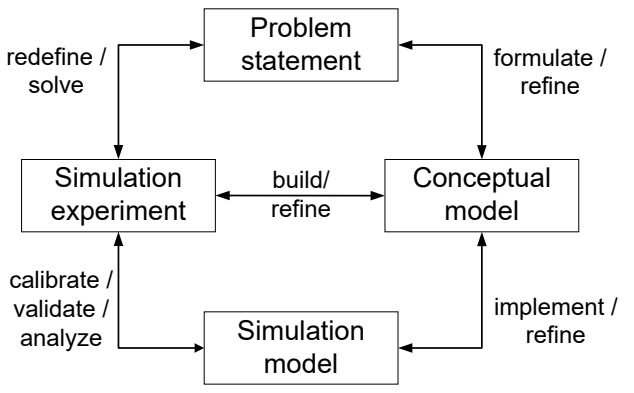

Fig. 1. Overview of the modeling and simulation life cycle with its main artifacts and activities.

pathway, virtual prototyping of a neurostimulator, and finite element analysis of electric fields).

The paper is organized as follows. In Section II, we provide an overview of related work. In Section III, we present the design of our novel model-driven approach for conducting simulation experiments. Details of the open-source implementation are given in Section IV. In Section V, we demonstrate the benefits of our approach using three real simulation studies. Finally, we end the paper with conclusions and future work in Section VI.

\section{RELATED WORK}

Model-driven approaches for modeling and simulation so far have mostly focused on the generation of (executable) simulation models [24]-[26], models in distributed settings [27], or multi-formalism modeling [28].

With regards to simulation experiments, MDE has been applied in the context of experiment design, and hypothesis testing. Teran-Somohano et al. [14] support the specification and execution of simple simulation experiments based on factorial designs, e.g., parameter sweeps. The approach by Dayıbaş et al. [29] is based on an own domain-specific language for experiment design and semi-automatic transformations to multiple execution platforms. Yilmaz et al. [15] propose the generation of experiment designs from hypotheses and outline an overarching goal-hypothesis-experiment framework.

Simulation experiments are also generated without explicit meta models or MDE frameworks. These usually target specific problems within the modeling and simulation life cycle. Lorig [30], for instance, generates efficient experiment designs for hypothesis testing based on formally specified hypotheses. Peng et al. [31] support the successive composition of models by generating simulation experiments for composed models. More specifically, statistical model checking experiments of individual models are reused and adapted for the composed model based on explicit experiment specifications and explicit behavioral properties. Similarly, properties of extended or refined models can be checked [32]. Cooper et al. [33] focus on supporting the comparison of electrophysiology models using typical experiment types of that domain, i.e., time course analyses and steady-state analysis. Concrete experiment specifications (protocols) conducted with previous models are stored in an online database and can be repeated with new models. Ruscheinski et al. [21] present a pipeline for 


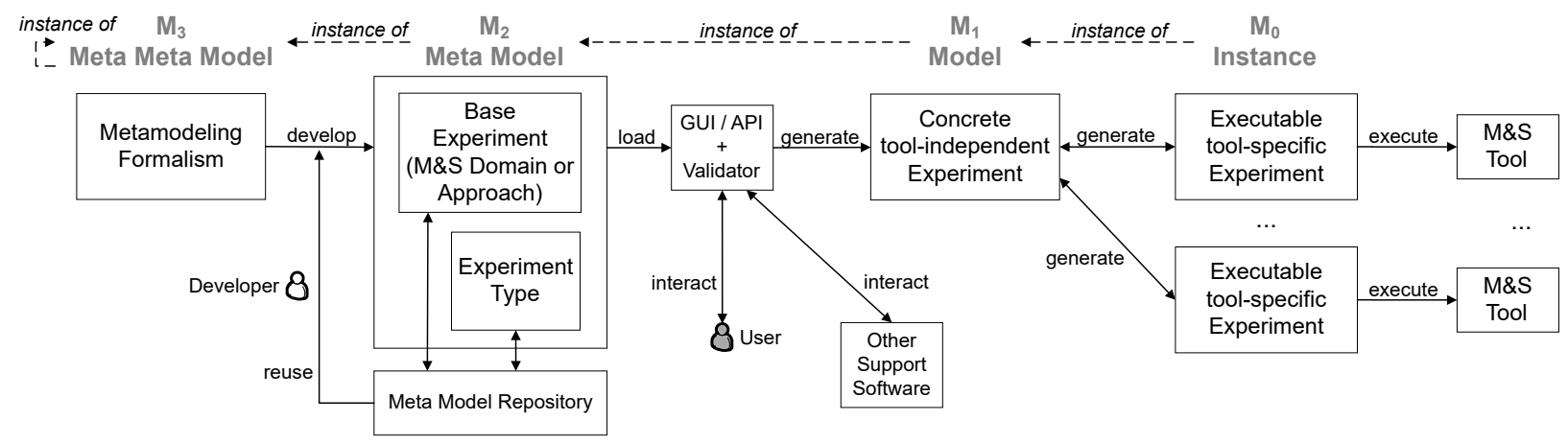

Fig. 2. Four-layered MDE architecture [23] for simulation experiments. The level of abstraction decreases from left to right. The experiment meta models (consisting of base experiment and experiment type, see layer $M_{2}$ ), developed based on a meta modeling formalism (layer $M_{3}$ ), are at the heart of the approach. Based on $M_{2}$, a tool-independent, exchangeable experiment specification can be generated $\left(M_{1}\right)$, which then forms the basis to derive an executable, toolspecific specification $\left(M_{0}\right)$ based on tool-specific mapping information. The generated experiment specification can then be automatically executed using a backend binding. Additional support components such as meta model repositories, graphical user interfaces, and validators help conducting simulation experiments effectively. The experiment generation pipeline may be used either manually, or integrated with other support software, for example, workflowbased, via an API.

generating a number of different experiment types including local sensitivity analysis, optimization, and statistical model checking. This pipeline was expanded by experiment schemas in Wilsdorf et al. [34] to support multiple simulation tools and approaches.

In this paper, we generalize the latter experiment generation approach further using the MDE paradigm. The main novelties are a) the separation into two types of meta models (domain meta models and experiment type meta models) and a corresponding composition mechanism, b) a meta model repository, c) bi-directional transformations and execution bindings for a variety of tools, and d) an application programming interface (API) for easy integration with other frameworks. As a result, our framework flexibly supports the conduction of diverse, complex experiment types, and it enables the sharing and reusing of knowledge even across different modeling domains and tools. Furthermore, our framework provides the foundation for automatically generating and reusing simulation experiments during the various phases of a simulation study, and thus for conducting entire simulation studies in a more systematic and effective manner.

The development of meta models, as part of an MDE framework, is closely related to the development of ontologies as both represent knowledge in a structured manner. Ontologies for modeling and simulation have been developed to facilitate data exchange, interoperability, and annotation of simulation resources [35]. Most ontologies define the components and concepts in simulation models instead of simulation experiments, e.g., the Discrete Event Simulation Component Ontology (DESC) [36], or the Discrete-event Modeling Ontology (DeMO) [37]. However, some ontologies overlap with meta models for simulation experiments, e.g., the ontology for capturing physics-based models by Cheong and Butscher [38] and our meta model for Finite Element Simulation Studies (see Sec. V) both include the boundary condition and material properties.

\section{MOdEL-DRIVEN APPROACH FOR CONDUCTING SIMULATION EXPERIMENTS}

We develop a novel model-driven approach for conducting simulation experiments. Its central features are the separation into two types of meta models (domain meta models and experiment type meta models) and a corresponding composition mechanism, a meta model repository, bi-directional transformations and execution bindings for a variety of tools, and an API for easy integration with other frameworks. The approach as a whole can improve knowledge sharing across domains and approaches, increase productivity and quality for complex experiments, make simulation experiments reusable, and automatically generate and execute simulation experiments in various settings.

\section{A. Framework Overview}

Our approach for supporting the conduction of simulation experiments is based on the MDE principle. The central idea of MDE is combining meta models (i.e., a structured representation of concepts) with means for code generation [39]. Typically, MDE is realized in an architecture with four levels of abstraction and translations between them [23]. Fig. 2 illustrates our four-level MDE approach for simulation experiments. Experiment meta models of the various domains and approaches of modeling and simulation are represented by the level $\left(M_{2}\right)$. In addition to the definition of these so-called base experiments, meta models for a variety of experiment types (e.g., sensitivity analysis (SA) or simulationbased optimization) exist. These two types of meta models can be composed flexibly to create meta models of complex simulation experiments. How meta models can be constructed is defined at the level of the meta meta model $\left(M_{3}\right)$, for example, one could use formalisms such as UML class diagrams [40] or schema languages [41], [42] to express the meta models. But experiment meta models are not only developed from scratch, therefore we store them in a repository from which parts can be reused by related areas of simulation or by other experiment types. The newly developed meta models 
are again stored in the repository and thereby complement the knowledge collection about simulation experiments. A composed meta model (consisting of base experiment and experiment type) can be loaded via an interface (GUI or API). The loaded meta model dictates which inputs have to be provided to specify a valid simulation experiment. An experiment validator provides guidance for the modeler. If all inputs required by the meta model have been collected, a concrete tool-independent experiment specification (model $M_{1}$ ) is generated. This general representation of the simulation experiment can now be easily reused, and some tools might also be able to import this specification directly. To receive an executable experiment instance $\left(M_{0}\right)$, the $M_{1}$ specification is automatically transformed to code of a target backend and subsequently executed using the respective tool binding. In the following, we will describe the distinct features of our framework further.

\section{B. Meta Modeling Language}

At the highest level of abstraction in our framework, the meta meta model is situated. The meta meta model is essentially the language in which the meta models are specified. Meta meta models are usually self-referential (i.e., they define themselves). As a result, no further layers above $M_{3}$ are required.

A meta modeling language needs to encompass means for comfortably developing a new meta model for a particular simulation domain and/or approach (base experiment), or a particular type of simulation experiment (experiment type). We have identified the following requirements:

- Hierarchical structuring via components and nested input properties;

- Unique names for components and input properties;

- Standard data types such as boolean, string, integer, real, as well as arrays;

- Enumeration of admissible values, and specification of default values;

- Maps to associate a key property with one or more other properties;

- Alternative specifications for input properties;

- Constraints to define simple type restrictions or the cardinality of an input property, and optionally relations between multiple input properties.

UML [40] is widely used for meta modeling software systems. UML class diagrams allow for a graphical notation that is intelligible to software developers as well as domain experts. The expressiveness of UML class diagrams can be complemented with the Object Constraint Language (OCL) [43]. Another way of meta modeling are schema languages like XML Schema [41] or JSON Schema [42]. They describe the structure of text documents, and therefore directly ship with a data exchange format (XML or JSON) for the layer $M_{1}$. They also encompass built-in means for defining simple constraints. For detailed syntax and semantics of the constraint languages, we refer the reader to the OCL specification and the XML/JSON Schema specifications.
Listing 1. A base meta model of a fictive modeling domain defined in JSON Schema.

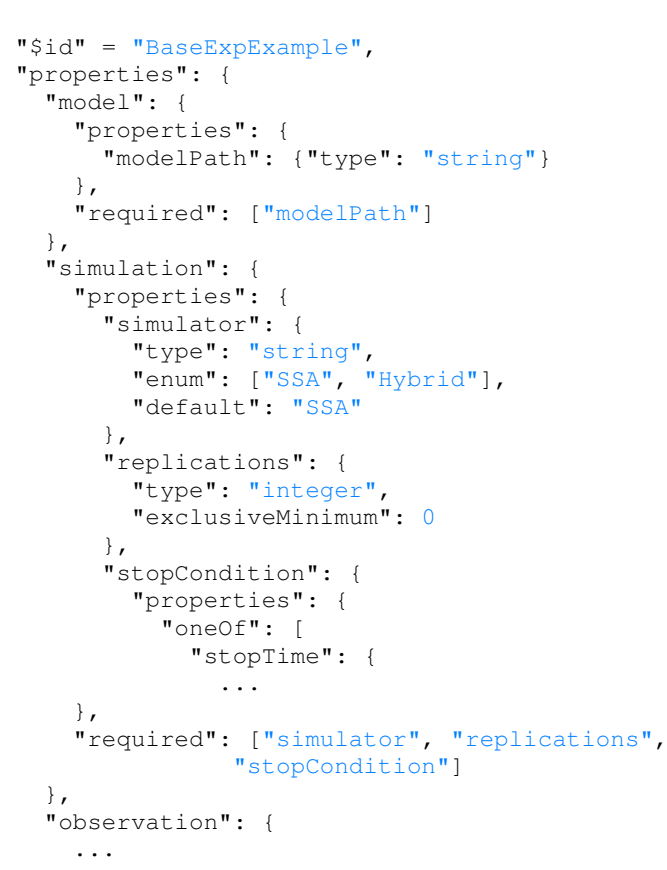

We found that both UML and JSON Schema are appropriate ways of specifying the experiment meta models. With the workings of our model-driven approach in mind, we decide to use JSON Schema in the following. However, if conversions between the different meta modeling languages exist, the actual choice of formalism becomes less important.

\section{Composition of Modeling Domains and Experiment Types}

The meta models describe the structure and ingredients of simulation experiment specifications. We distinguish base meta models and experiment type meta models. Base meta models describe simple experiments (i.e., runs with single parameter configurations) in a specific domain or modeling approach. Meta models for experiment types, on the other hand, allow for supporting complex experiments where parameters are varied and specific analyses are conducted on the outputs.

A base meta model for a fictive domain can be seen in Listing 1 (meta models for experiment types can be created analogously). The meta model is defined using JSON Schema and contains all the necessary information in one, hierarchically structured file. It structures simulation experiments of the fictive domain into a model component, a simulation component, and an observation component. Each component encloses various other input properties, characterized by type information, choices, default values, and constraints. For example, the constraint exclusiveMinimum: 0 was added inside the property replications. To express which inputs are required for specifying a valid simulation experiment, the keyword required can be used. Also, default values can be expressed explicitly, e.g., for setting SSA (stochastic simulation algorithm) as the standard simulator type of the domain. Moreover, to express alternatives the keyword oneof 
is used, as in the case of stopcondition, which can be given by a) a specific stop time, or b) an expression on the model output. To build a valid simulation experiment that conforms to the meta model, exactly one of these alternatives needs to be applied. However, to keep the example short we do not specify these options further and omit details of the observation component.

For specifying a simulation experiment at least a base meta model needs to be selected and filled with concrete input values. However, the base meta models can also be flexibly composed with the various meta models for experiment types to create a meta model for the current experimentation task at hand. Using the composition mechanism, complex simulation experiments (such as statistical model checking, parameter estimation, steady state analysis, etc.) can be supported for any modeling approach or domain. This is possible due to the orthogonality of the base experiment specification and the experiment type specification. Note that in this paper we focus on the main experiment types and their methods, and do not discuss further analyses (i.e., post-processing or plotting of the results). However, this could be added as another component in the meta models. The language SED-ML [9], for instance, allows the specification of various plot types including axis labels, etc.

\section{Meta Model Repository}

The developed meta models are stored in a model repository. This gives users (modelers) access to a variety of meta models that they can flexibly compose, as discussed earlier. For developers, the existing meta models contained in the repository can be the starting point for developing new ones. Often, depending on the needs of the new domain or approach, not everything has to be developed from scratch, but meta model components can be exchanged, added, or modified. Here, the reuse of knowledge about simulation experiments is not limited by specific domains and approaches. For example, research in the context of discrete-event simulation [5], [7] and computational biology [44] has identified a few common constituents of basic simulation experiment specifications: model configuration, simulation initialization, and observation. With respect to experiment types, meta models can be extended to include more elaborate methods, e.g., by new sampling strategies or distance measures.

\section{E. Interfaces}

Our pipeline can be used in two ways. The first option is a dynamic graphical user interface (GUI), which we provide with our framework. Depending on the selected base meta model and the experiment meta model, a tailored GUI is generated to support modelers in specifying their simulation experiments. Fig. 3 shows a screenshot of a GUI generated from the meta model example shown in Listing 1, and a meta model for SA. In the GUI the meta model components are displayed as individual tabs ("Model", "Simulation", "Observation"), input properties as rows, and alternative definitions are selected via drop-down menus where, depending on the selection, other input properties become available as in the

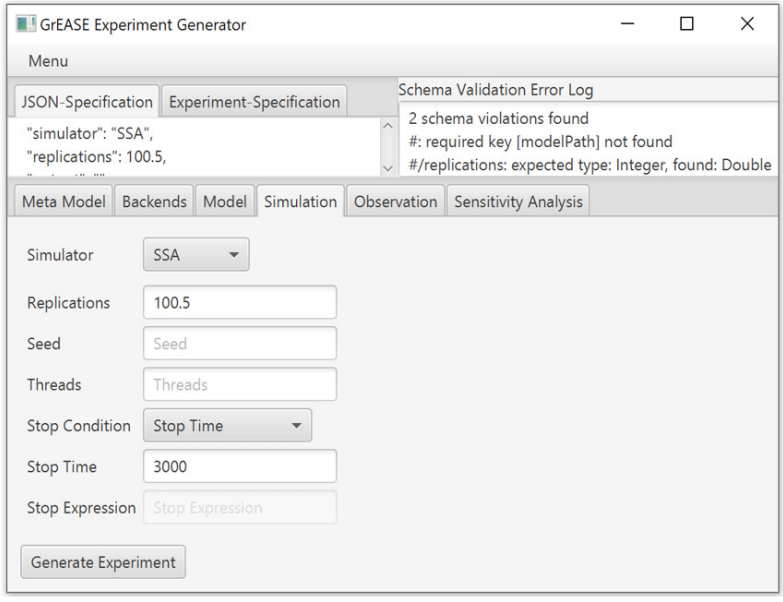

Fig. 3. Screenshot of the experiment generation GUI. For a given meta model, and depending on the provided inputs, new input fields are generated. Validation errors are reported at the top right if the inputs do not conform to the meta model. The generated experiment specifications (tool-independent and tool-specific) are displayed at the top left.

case of "Stop Condition". The selection of the domain or approach and the experiment type takes place in the tab "Meta Model". Thereby, an additional tab for the experiment type "Sensitivity Analysis" was created (Fig. 3). The tab "Backends" is used to select the code generation target.

As the second option, we provide an API. It allows flexible integration of our model-driven framework with other software. Via the API, the meta models can be selected and composed, the experiment inputs can be passed and validated, the GUI can be opened, the target backend can be chosen, and experiment code can be generated or parsed. Consequently, one could integrate our framework with a procedure that automatically extracts certain experiment inputs from the model documentation (parameter tables or conceptual model) [20], [21], or automatically generates simulation experiments from inside a workflow system (see Sec. V). If not all input fields dictated by the meta model can be filled automatically, the simulation experiment can be completed manually through the user interface.

\section{F. Experiment Validation}

Before inputs are passed on to the next layer $\left(M_{1}\right)$ to produce a concrete tool-independent experiment specification, the entered values have to be validated. The validator checks conformance with the chosen meta models, and thus both structural checks and type checks are carried out. After each validation cycle, the validator gives immediate feedback to the user (see Fig. 3) or the application, depending on which interface is used for the interaction. This step-by-step guidance supports inexperienced users, but also experienced users can benefit, as they do not have to concern themselves with the intricate details of simulation experiment specifications, and instead can concentrate on important tasks such as output analysis and result interpretation.

Listing 2 shows a created JSON document at the layer $M_{2}$. The document is validated according to the meta model 
Listing 2. Simulation experiment filled according to the JSON meta model defined in Listing 1. Validation errors in the specification are marked red.

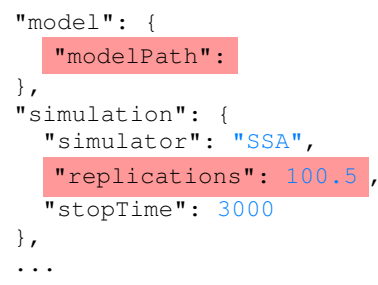

example described above. The validation is unsuccessful due to the missing property modelPath and due to using the wrong data type for the input property replications.

\section{G. Transformations and Bindings}

The experiment meta models provide a general vocabulary that all modeling and simulation tools can refer to and communicate with. Meta models are therefore one way for improving the interoperability of modeling and simulation software. To go from an abstract experiment specification in a tool-independent format to executable code, the meta models have to be mapped to the syntax of actual modeling and simulation tools. During this transformation step, also differences in the terminology need to be resolved. E.g., the concept of the stochastic simulation algorithm (SSA) has numerous implementations and is therefore known under different names such as NextReactionMethod () or GibsonBruck () method.

Often one wants to generate code for a single tool and thus in a single language. However, it is not uncommon to run the simulations in one tool, collect the results, and then run the analysis in another. Our transformation mechanism supports the combination of tools for simulation and analysis, and thus allows generating a combination of two scripts in two different languages. Moreover, for certain experiment types, such as SA, the toolchain can be divided further (see Section V-B).

Another important feature of the transformations is their bidirectionality. This means that we can (backward) parse a concrete experiment specification of a specific language and represent it in the canonical format. From there, forward transformations can generate the same experiment for a different tool, or the experiment may be reused and adapted for a different purpose.

Although it seems arduous to implement a transformation, it is far more efficient than to connect all pairs of tools individually. Moreover, once agreed upon in the community, the structure and vocabulary of a meta model is persistent and will rarely change but rather be extended with new content which will lead to some extensions in the transformations. It might also be beneficial to maintain transformations to distinct versions of the same M\&S tool or to legacy systems for keeping older simulation experiments reproducible and, for example, for testing the tool itself. In addition, the experiment meta models provide guidelines for implementing new tools and therefore promote a more structured approach for developing modeling and simulation software.

In addition to the code transformations, we maintain socalled bindings to the various tools for which code is generated. The bindings allow us to automatically run the generated experiment specifications with the chosen backend. Note that a suitable backend could also be chosen automatically for a given task since the transformations make explicit which experimentation methods are implemented where.

\section{IMPLEMENTATION}

Our model-driven framework for conducting simulation experiments is realized in Java 1.8. This proof-of-concept implementation, thus far, comprises meta models for the three simulation domains and different experiment types, as well as transformations and bindings to several backends (see Evaluation). The source code is publicly available in a Git repository ${ }^{2}$.

At the heart of the implementation are the simulation experiment meta models which are implemented using JSON Schema, Draft-07 [42]. We selected JSON Schema as a basis to formulate our schemas, since over the past years JSON has probably become the most popular format for exchanging data on the web, and therefore a variety of capable parsers and validators exist. For a selected schema, our dynamic GUI, implemented using JavaFX, generates the necessary input tabs and fields (as shown in Fig. 3). All collected user inputs are stored in the multipurpose data interchange format JSON [45]. The JSON files are validated against the JSON schemas using an open-source JSON Schema validator for Java, based on the org.json API [46].

For the mapping between the general schema inputs and the syntax of actual modeling and simulation tools, we use the template engine FreeMarker [47]. FreeMarker has a built-in template language in which the mappings can be specified. For each backend and schema input property, a mini template is implemented. A master template joins all the template snippets together. The template engine takes this master template and fills the template variables with the data from the JSON file.

\section{Evaluation}

Applying MDE for simulation experiments has positive effects on the building and sharing of knowledge, the productivity and quality of code, the reusability, as well as the automatic generation of simulation experiments. This will ultimately lead to entire simulation studies being conducted in a more effective and systematic manner.

We demonstrate the benefits using three case studies from the context of electrically active implants (stochastic discreteevent simulation of a cell signaling pathway, virtual prototyping of a neurostimulator, and finite element analysis of electric fields). We believe that well-designed examples are the best way to convince modelers to adopt MDE of simulation experiments in their daily practice and to integrate this approach with other toolchains.

\footnotetext{
${ }^{2}$ https://git.informatik.uni-rostock.de/mosi/exp-generation
} 
TABLE I

BASE META MODEL FOR CONDUCTING EXPERIMENTS USING STOCHASTIC DISCRETE-EVENT SIMULATION. TO CREATE A VALID SIMULATION EXPERIMENT, VARIOUS INFORMATION ABOUT THE MODEL, THE SIMULATION, AND THE OBSERVED QUANTITIES HAS TO BE PROVIDED. EACH ROW DESCRIBES AN INPUT PROPERTY OF THE META MODEL. SUB-PROPERTIES ARE DENOTED BY “•”. ALTERNATIVE META MODEL PARTS ARE INDICATED BY “ $\rightarrow$ ".

\begin{tabular}{|c|c|c|c|c|c|c|}
\hline & Name & Description & Type & Choices & Default & Required \\
\hline \multirow{7}{*}{$\frac{\bar{d}}{\frac{d}{2}}$} & modelFile & Specify the simulation model & Alternative & - & - & yes \\
\hline & $\rightarrow$ folder & Folder of the simulation model & String & - & - & yes \\
\hline & fileName & Name of the simulation model & String & - & - & yes \\
\hline & $\rightarrow$ reference & Reference to the simulation model & String & - & - & yes \\
\hline & configuration & Configure the model parameters & Map & - & - & no \\
\hline & •parameterName & Input parameter name (key) & String & - & - & no \\
\hline & •parameterValue & Input parameter value & Real & - & - & no \\
\hline \multirow{7}{*}{ 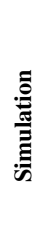 } & simulator & Choose simulation algorithm & String & SSA, Hybrid, ... & SSA & yes \\
\hline & replications & Number of simulation replications & Integer, $>0$ & - & 1 & yes \\
\hline & randomSeed & Initialize random number generation & Long, $>0$ & - & - & no \\
\hline & parallelThreads & Number of parallel threads & Integer, $>0$ & - & 1 & no \\
\hline & stopCondition & Type of stop condition & Alternative & - & - & yes \\
\hline & $\rightarrow$ stopTime & Stop at specific point of time & Real, $>0$ & - & - & yes \\
\hline & $\rightarrow$ stopExpression & Stop based on simulation state & String & - & - & yes \\
\hline \multirow{7}{*}{ 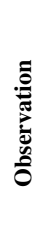 } & observables & Specify the observables & Map & - & - & yes \\
\hline & •observationExpression & Expression on model entities (key) & String & - & - & yes \\
\hline & •observationAlias & Alias for observation expression & String & - & - & no \\
\hline & observationTime & Choose option for observation & Alternative & - & - & yes \\
\hline & $\rightarrow$ observationTimes & Observe at specific points of time & Array $<$ Real $>,>0$ & - & - & yes \\
\hline & $\rightarrow$ observationRange & Observe time range and interval & Array $<$ Real $>$, length $=3$ & - & - & yes \\
\hline & outputFormat & Choose reporting format & String & $\mathrm{CSV}, \ldots$ & - & no \\
\hline
\end{tabular}

In the following, we first develop meta models to capture the characteristics of the base simulation experiments of these three modeling domains and discuss how knowledge about simulation experiments can be exchanged within, but also between the different modeling domains and approaches. Next, we demonstrate that, based on the base meta models composed with a shared meta model for the experiment type "global sensitivity analysis", three complex simulation experiments can be specified in a straightforward manner. Then, we show how our JSON-based format can function as a standard exchange format to facilitate the automatic reuse of simulation experiments, e.g., for the cross-validation of two related models. Finally, we show how our approach can be used to automatically generate simulation experiments by extracting information from a workflow.

Note that all the experiments we describe are only snapshots of the three studies in the form of single simulation experiments. During the simulation studies, of course, further analysis steps with the same or other experiment types (e.g., simulation-based optimization or statistical model checking) are involved, until the initial research questions can be answered. For these further steps, our approach can be applied analogously.

\section{A. Improved Knowledge Sharing across Domains}

Our three case studies are conducted in the context of electrically active implants. However, they focus on different aspects involved in the development of such implants and therefore require different modeling and simulation approaches. Consequently, they require different meta models for the conduction of their basic experiments. In this section, we introduce meta models for these approaches, i.e., the base experiments for stochastic discrete-event simulation, virtual prototyping of heterogeneous systems, and finite element analysis in electromagnetics. This demonstrates the versatility of our approach, and — most importantly—its value for improving knowledge sharing within and across the diverse domains and approaches of modeling and simulation.

1) Meta Model for Stochastic Discrete-Event Simulation: Stochastic discrete-event simulation (DES) is applied for modeling systems where the variables change at discrete time points, and the time of the next event is determined stochastically [48], [49]. In cell biology, e.g., modeling and simulating stochastic effects is of significant interest, especially for processes that involve low copy numbers [50].

Table $\mathrm{I}^{3}$ shows the developed DES meta model which comprises three essential components, i.e., model configuration, simulation initialization, and observation (represented by sections in the table). In the meta model, each component of a simulation experiment requires specific inputs (table rows). For instance, typical ingredients for a stochastic simulation are now made explicit, such as the number of replications, the random seed, and the number of parallel threads (see simulation component). Each input is characterized by a unique name, a description, a data type, a set of choices, a default value, and information about whether this input is required or optional (table columns). Properties of type Map (e.g., configuration) assemble related inputs as keyvalue pairs. The assembled sub-properties are indicated by “•”. Other properties (e.g., model, stopCondition, or

${ }^{3}$ For the paper, we use tables to represent the meta models in a compact and readable way. The implementation as JSON Schema documents can be viewed in our source code. 
TABLE II

MODIFIED META MODEL COMPONENT FOR VIRTUAL PROTOTYPING OF HETEROGENEOUS SYSTEMS. IN CONTRAST TO SIMULATIONS THAT ARE ONLY BASED ON DISCRETE EVENTS, HERE ALSO A TIME STEP HAS TO BE CONFIGURED FOR THE NUMERICAL INTEGRATION. THE ROWS DESCRIBE THE DIFFERENT INPUT PROPERTIES OF THE META MODEL. SUB-PROPERTIES ARE DENOTED BY “•”. ALTERNATIVE META MODEL PARTS ARE INDICATED BY " $\rightarrow$ ".

\begin{tabular}{|c|c|c|c|c|c|c|}
\hline & Name & Description & Type & Choices & Default & Required \\
\hline \multirow{9}{*}{ 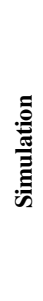 } & replications & Number of simulation replications & Integer, $>0$ & - & 1 & yes \\
\hline & randomSeed & Initialize random number generation & Long, $>0$ & - & - & no \\
\hline & parallelThreads & Number of parallel threads & Integer, $>0$ & - & 1 & no \\
\hline & stopCondition & Type of stop condition & Alternative & - & - & yes \\
\hline & $\rightarrow$ stopTime & Stop at specific point of time & Real, $>0$ & - & - & yes \\
\hline & $\rightarrow$ stopExpression & Stop based on simulation state & String & - & - & yes \\
\hline & timeStep & Time step of the simulator & Map & - & - & yes \\
\hline & •timeStepSize & Size of time step & Real & - & - & yes \\
\hline & •timeStepUnit & Unit of time step & String & $\mathrm{s}, \mathrm{ns}, \ldots$ & - & yes \\
\hline
\end{tabular}

observationTime) are of type Alternative and provide different ways of specifying a property. The different options are indicated by " $\rightarrow$ ". For instance, the simulation model can be provided by either a folder and filename (local files) or a reference to an online resource.

The above meta model generalizes the structure and ingredients of simulation experiments at the level of the modeling and simulation approach (i.e., DES). It can therefore be used for supporting the specification and execution of basic simulation experiments in various modeling domains, such as cell biology or digital circuits.

2) Meta Model for Virtual Prototyping of Heterogeneous Systems: Virtual prototyping allows for the design and development of products via modeling and simulation where building real prototypes is infeasible, e.g., due to ethical concerns, as in the case of neurostimulators. The fundamental paradigm for modeling and simulation of digital circuits is DES. This means that the knowledge about the ingredients of simulation experiments captured in the DES meta model can be applied there as well. However, some virtual prototypes include components outside of the digital domain (e.g., to model the voltage levels of a battery) and thus require continuous-time representation. For these models, using the DES meta model for the simulation experiments does not suffice. However, using our framework, we can, with relatively low effort, define a new experiment meta model for virtual prototyping of heterogeneous systems based on the existing DES experiment meta model. The model configuration component and the observation component can be reused from the shared meta model repository as they are. Only the simulation initialization component needs to be adapted for the new simulation approach (see Table II). The main difference is that now a fixed time step and time step unit are included, at which the discrete-time and continuous-time models are synchronized. Furthermore, the modified meta model does not include the type of solver or scheduler explicitly as this is usually assigned automatically to specific semantics defined in the language standard (e.g., SystemC-AMS [51]). Thus, the solver or scheduler is part of the simulation model and not changed in experiments.

3) Meta Model for Finite Element Analysis in Electromagnetics: Finite element analysis (FEA) is a general method that is capable of treating complex geometries and accurately computing, e.g., the properties and effects of electric fields in deep brain stimulation. As FEA is a completely different modeling and simulation approach, no parts from the previously defined base meta models can be reused, and a new one is developed. Due to the separation in geometric model and physical model, the experiment meta model for FEA (see supplementary material, Table 1) has two new components: geometric model and physical model. These are complemented by a simulation component that comprises information about the type of solver, and accuracy of the solution (given by the coarseness of the mesh). For the observation component, derived quantities can be specified as well as coordinates at which to evaluate these quantities.

Note that this first draft of the FEA experiment meta model was developed with the background of electromagnetics in mind. Future efforts should aim to support FEA more generally. In particular, the various inputs and constraints depending on the type of physics need to be identified. In addition, multiphysics applications where mechanics, electromagnetics, and thermodynamics are coupled are of potential interest. In each discipline, PDEs comprising material properties together with geometrical constraints are the basis of the modeling approach [52]. Hence, the current meta model can provide a basis for further discussions and adjustments.

\section{B. Increasing Productivity and Quality for Complex Experi- ments}

Besides sharing information about the base experiments, our approach is designed to share meta models for diverse, complex experiment types. Table III, for instance, shows a meta model for global SA, that could be added to any of the above-described base meta models. It comprises various important ingredients for the specification of global SAs, such as factor ranges and factor distributions, as well as a choice of different sampling strategies: Monte Carlo $(M C)$ and Quasi-Monte Carlo (QMC) [53], Orthogonal Latin Hypercube $(O L H C)$ [54], and Polynomial Chaos expansion (PC) [55]. For the first three strategies, the samples are used directly to calculate the indices. With PC on the other hand, first a surrogate model is constructed based on which the indices are computed. As index type, e.g., Sobol indices [56], [57] can 
TABLE III

META MODEL FOR THE EXPERIMENT TYPE “GLOBAL SENSITIVITY ANALYSIS". TO CREATE A VALID SENSITIVITY ANALYSIS EXPERIMENT, VARIOUS INFORMATION ABOUT THE MODEL FACTORS HAS TO BE PROVIDED AS WELL AS INFORMATION ABOUT THE SAMPLING PROCEDURE (EXPERIMENT DESIGN), AND INSTRUCTIONS FOR CALCULATING THE SENSITIVITY INDICES. THE ROWS DESCRIBE THE DIFFERENT INPUT PROPERTIES. SUB-PROPERTIES ARE DENOTED BY “•”. ALTERNATIVE META MODEL PARTS ARE INDICATED BY “ $\rightarrow$ ”.

\begin{tabular}{|c|c|c|c|c|c|c|}
\hline & Name & Description & Type & Choices & Default & Required \\
\hline \multirow{10}{*}{ 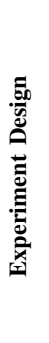 } & factors & Information about the model factors & Map & - & - & yes \\
\hline & •factorName & Name of the factor (key) & String & - & - & yes \\
\hline & •factorMinimumValue & Lower bound on the factor value & Real & - & - & yes \\
\hline & •factorMaximumValue & Upper bound on the factor value & Real & - & - & yes \\
\hline & •factorDistribution & Assumed distribution of the factor & String & Uniform, Normal, ... & Uniform & yes \\
\hline & •factorDistributionParameters & Parameterize the distribution & Map & - & - & no \\
\hline & •distributionParameterName & Parameter of the distribution (key) & String & - & - & no \\
\hline & •distributionParameterValue & Initialize the distribution parameter & Real & - & - & no \\
\hline & samplingStrategy & Choose the sampling strategy & String & $\mathrm{MC}, \mathrm{QMC}, \mathrm{OLHC}, \mathrm{PC}, \ldots$ & $\mathrm{MC}$ & yes \\
\hline & sampleSize & Number of samples & Integer & - & - & no \\
\hline \multirow{2}{*}{ 弟 } & indexType & Choose type of sensitivity index & String & Sobol, ... & - & yes \\
\hline & bootstrapCI & Calculate confidence interval with bootstrapping & Boolean & - & false & no \\
\hline
\end{tabular}

be used, which are variance-based measures. The first-order Sobol index of factor $x_{i}$ describes the individual contribution of this factor to the overall variance in the output $V(y)$ :

$$
S_{i}=\frac{V_{x_{i}}\left[E_{\boldsymbol{x}_{\sim i}}\left(y \mid x_{i}\right)\right]}{V(y)} .
$$

The total-order sensitivity index, $T_{i}$, accounts for all the contributions to the output variation due to factor $x_{i}$ (i.e., firstorder index plus higher-order interactions):

$$
T_{i}=\frac{E_{\boldsymbol{x}_{\sim i}}\left[V_{x_{i}}\left(y \mid \boldsymbol{x}_{\sim i}\right)\right]}{V(y)} .
$$

Having made explicit the ingredients of global SA as a meta model, we can easily specify simulation experiments to calculate Sobol indices for various simulation models. We show this for three different models by composing the respective base meta model with the SA meta model. This has the potential to increase productivity during the simulation study, since guidance is provided via a specialized GUI and input validation. Moreover, complicated details of the code are abstracted away by the model-driven approach. Thus, the modeler does not have to worry about how to combine the simulation tools and analysis tools in a complex experiment. Fig. 5 shows three different ways of performing a Sobol analysis - they all can be generated using the same meta model.

1) Sensitivity Analysis of a Wnt Signaling Model: To understand how electrically active implants affect the differentiation and proliferation of cells (and thus the composition and regeneration of tissue), the impact of external electric fields on central cellular signaling pathways needs to be studied. The $W n t / \beta$-catenin signaling pathway is one of the central pathways that regulate proliferation as well as differentiation of cells [58]. Deregulated forms of this pathway are involved in several human cancers and developmental disorders [59]. Effects on membrane-related dynamics are of particular interest for the development of electrically active implants. Lipid rafts, specialized microdomains of the membrane, have been found to sense the electric field and to direct cellular responses of cells [60]. Therefore, a Wnt simulation model [61] has been extended to capture both raft- and non-raft-associated endocytic processes of the $\mathrm{Wnt} / \beta$-catenin receptor LRP6 in detail, including stochastic effects [62].

The model ${ }^{4}$ is written in ML-Rules [63] and simulated using stochastic DES. Therefore, the experiment meta model for DES can be used to guide through the specification of the base experiment. Then, the modeler is guided through the definition of the global SA. There, the modeler is interested in the global sensitivity of the observed quantity, which is the fraction of the cell membrane receptor LRP6, with regard to the parameters $k e_{\text {nonraft }}$ and $k e_{\text {raft }}$, which represent the internalization rates of bound LRP6 receptor complexes, and the parameter $k L R A s s$. Furthermore, the modeler specifies uniform distributions for the factors as well as minimum and maximum values. As the sampling strategy, an OLHC design with 1750 samples is selected.

Once all inputs for the SA have been collected, executable experiment code can be generated, i.e., a combination of an $\mathrm{R}$ script with a SESSL script, as illustrated in Fig. 5A. Since we have implemented bindings to both SESSL and R, the composed experiment can also be automatically executed. Fig. 4A presents the results of the experiment. They show almost no impact of the parameter $k e_{\text {raft }}$ on the results. The variances of each of the other two parameters $\left(k e_{\text {nonraft }}\right.$ and $k L R A s s$ ) make up about half of the variance of the result.

2) Sensitivity Analysis of a Battery Model: Deep brain stimulation is a therapy option for a multitude of neurological disorders. While it is widely implemented into the clinical routine especially for Parkinson's disease and Dystonia, the underlying mechanisms are not fully understood. Since most experiments cannot be performed directly on humans due to ethical reasons, animal testings are necessary. However, rodent-specific implants require a highly optimized level of miniaturization and power consumption compared to human implants. Physical prototyping is not feasible as the pursued runtime is more than a year. Therefore, virtual prototyping is

\footnotetext{
${ }^{4}$ The Wnt model, the SA experiment, and the analysis results are available at https://github.com/SFB-ELAINE/Case-Study-Endocytosis
} 
A

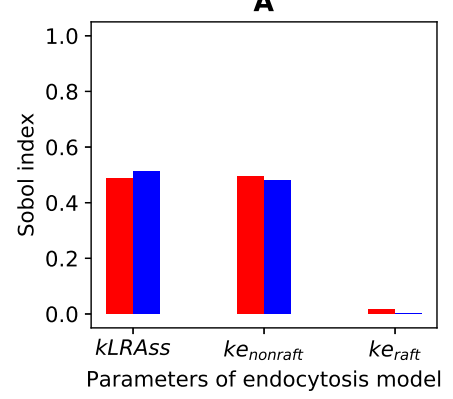

B

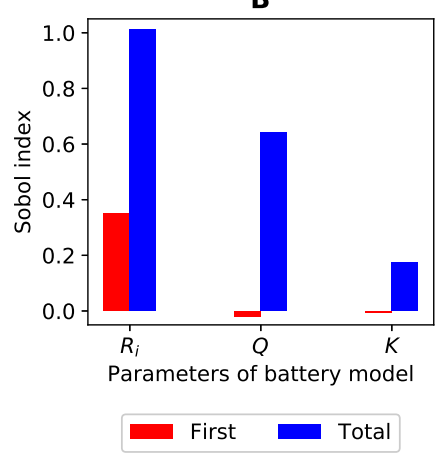

C

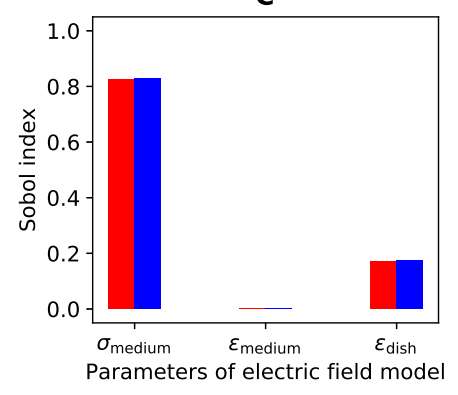

Fig. 4. First- and total-order Sobol indices calculated for the three models. Whereas the Wnt model (A) and the electric fields model (C) show linear behaviors, the difference between the first and total order values of the battery model (B) indicates that the behavior of the model is governed by the interaction of parameters.

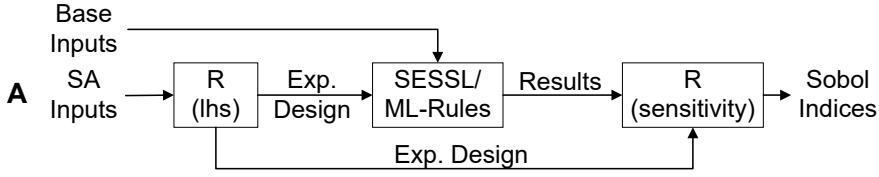

B
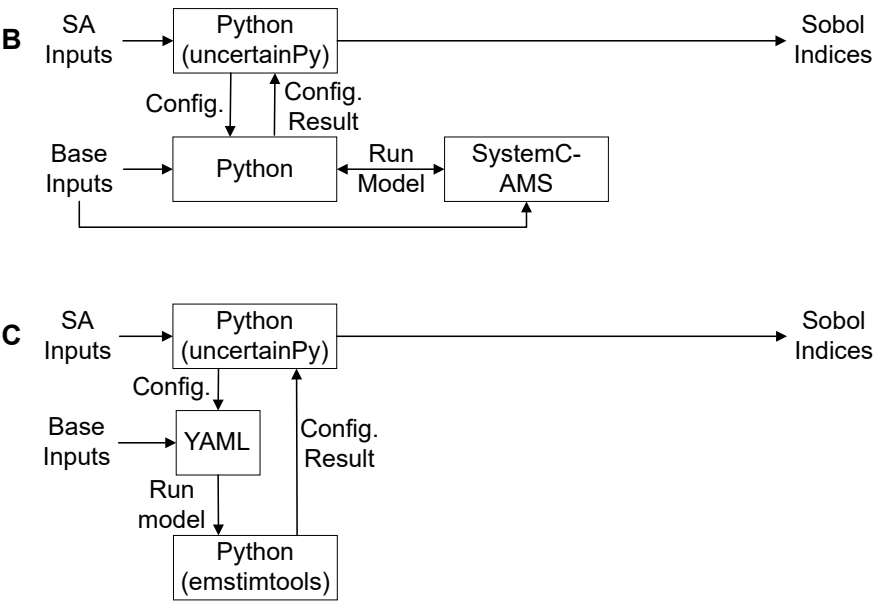

Fig. 5. Schematic of the generated code for the three sensitivity analysis (SA) experiments. The experiments take the user-given base inputs and SA inputs, and produce Sobol indices as outputs.

used to design neurostimulators for rodents [64]. The model ${ }^{5}$ considers the interplay between various heterogeneous system components (i.e., battery, boost converter, microcontroller, and stimulation unit) and thus combines digital as well as analog components. Whereas previous studies focused on the optimization of voltage levels inside the system, this study focuses on understanding the implications of different battery parameters on the overall runtime.

As they vary over different battery types, the internal resistance $\mathrm{Ri}$, the battery capacity $\mathrm{Q}$, and the polarization constant $K$ are of special interest. To facilitate the analysis of these parameters, the modeler uses the model-driven framework and

\footnotetext{
${ }^{5}$ The described SA experiment and the analysis results are available at https: //github.com/SFB-ELAINE/Case-Study-Neurostimulator. Unfortunately, the battery model itself cannot be provided, as it is part of a closed-source project.
}

selects the meta model for global SA (Table III) to enhance an already specified base experiment. First, parameter ranges and Gaussian distributions are specified for the three factors, and a Quasi-Monte Carlo sampling is selected with 1000 samples. A schematic of the generated code, based on the Python package UncertainPy [65] and SystemC-AMS [51], is shown in Fig. 5B. The generated experiment is started automatically, however, due to the complexity of the model, even after 12 hours, the analysis did not converge. Therefore, the experiment was terminated to find a more efficient approach. With the assistance of the model-driven framework, the sampling strategy could easily be substituted by Polynomial Chaos expansion (PC), and the experiment was automatically repeated. Using PC, the runtime could be reduced to less than 3 hours. The SA results are depicted in Fig. 4B and show that the model response is strongly determined by interactions between the parameters. The internal resistance seems to have a particularly strong effect on the overall battery runtime. Consequently, one should aim for measuring this parameter in (real) experiments and pre-select batteries with a lower internal resistance.

3) Sensitivity Analysis of an Electric Fields Model: The third simulation study aims to compute the electric field distribution in a specific chamber [66], [67]. Based on the computed field distributions, the biological response of the stimulated biological sample can be linked to certain specifications of the electrical stimulation set-up. A global $\mathrm{SA}^{6}$ is used to evaluate the influence of the dielectric parameters on the electric field strength at specific locations of the cells.

Following the structure of the meta model, the modeler enters all the required information (i.e., various material properties as factors and their value ranges) and assumes uniform distributions. Polynomial Chaos expansion is chosen as the sampling method, as is an often applied method in FEA where simulation runs are computationally expensive. Fig. 5C shows the generated code which combines the Python package EMStimTools [68] with UncertainPy [65]. The firstand total-order Sobol indices are shown in Fig. 4C. The results

\footnotetext{
${ }^{6}$ The model, the described SA experiment, and the analysis results are available at https://github.com/j-zimmermann/EMStimTools/tree/master/examples/ experimentSchemas
} 
Listing 3. Cross-validation experiment in the exchangeable JSON format, with the inputs (blue) already adapted for the Haack et al. model.

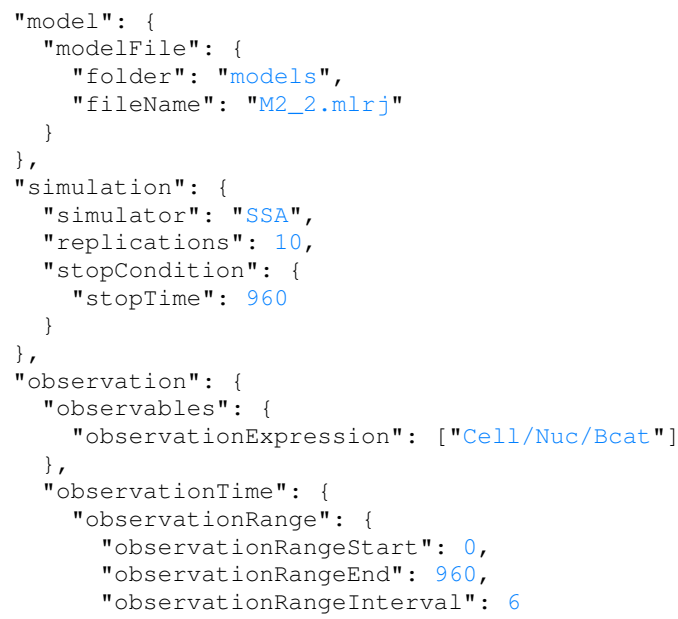

suggest that a change in the permittivity of the medium does not influence the field strength. Hence, the permittivity can be neglected in future uncertainty analyses for this kind of problem and similar input parameters.

\section{Reusability}

The final Wnt model (introduced in Sec. V-B1) is the result of successively extending simpler model versions. The original Wnt model by Lee et al. (2003) [69] was extended by raftand redox-dependent signaling events in a study by Haack et al. (2015) [61]. This new model was then extended further by endocytic processes in Haack et al. (2020) [62]. To ensure that the basic model behavior was not changed due to the extensions, various cross-validation experiments are required that compare the trajectories of the variables of interest.

Therefore, the original simulation experiments of the Lee et al. study shall be reused and repeated with the extended model. However, the original experiments were specified in SED-ML [9] and the corresponding model in SBML [70] (see BioModels [71] entry ${ }^{7}$ ). In contrast, the Wnt model by Haack et al. (2015/2020) was specified using the rule-based modeling language ML-Rules [63], and the experiments are conducted using the experiment specification language SESSL [7]. Thus, in order to replicate results from the Lee study, the SEDML experiment specification needs to be adapted for the new model, and translated to SESSL. This can be supported by the model-driven approach.

First, a meta model for DES directs the automatic parsing of the original specification and provides meaning to the parts of the experiment specification. As the experiment type is a time course analysis, a base meta model suffices to capture all the inputs. Once transformed to the quasi-standardized JSON format, the experiment specification can be adapted to work

${ }^{7}$ https://www.ebi.ac.uk/biomodels/BIOMD0000000658

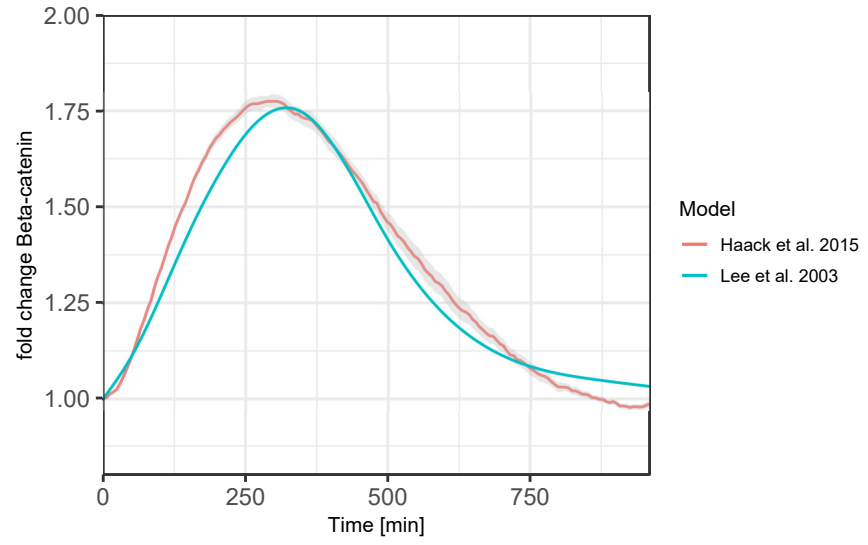

Fig. 6. Results of the cross validation of the Lee et al. model and the Haack et al. model (after applying a scaling factor of 0.28 ) to the $\beta$-catenin trajectory.

with the Haack model. E.g., the file name and the simulation stop time (due to the use of different time scales) need to be changed. To perform the adaptions automatically, for some experiment parts additional knowledge in the form of ontologies is required. E.g., UniProt [72] provides a unique identifier for each protein and allows to transform the observation expressions from one model to another. Listing 3 shows the experiment specification in the JSON-based exchange format, after being adapted for the Haack model.

After the adaptions are completed, the SESSL-specific experiment can be generated and executed automatically. The result of the cross validation is depicted in Fig. 6. It compares the trajectories of the key protein $\beta$-catenin, an indicator of the pathway's activity, produced by the Lee and the Haack model (with adapted time scale) when stimulated with a transient Wnt stimulus. Both $\beta$-catenin curves show the same peak at the same time. This means that the extensions applied in the study by Haack et al. do not alter the central dynamics of the pathway.

\section{Automation}

In the previous subsection, we already saw one case of automation, i.e., we demonstrated that a well-designed MDE pipeline is the foundation for reusing simulation experiments automatically [22] and allows integrating with ontologies. Now we go one step further and show that we can integrate this approach with other frameworks for supporting simulation studies as a whole, e.g., artifact-based workflows.

In an artifact-based workflow, the central products of simulation studies are identified and made explicit as artifacts. These include the conceptual model, the simulation models, and the simulation experiments. Each artifact is characterized by stages a modeler can move through to achieve certain milestones, and preconditions called guards [4]. Fig. 7 shows the conceptual model artifact of an artifact-based workflow for finite element studies [73]. While moving through the stages of the conceptual model, meta-information about the model is collected, such as the modeling objective, requirements, and input data. The meta-information collected inside of the 


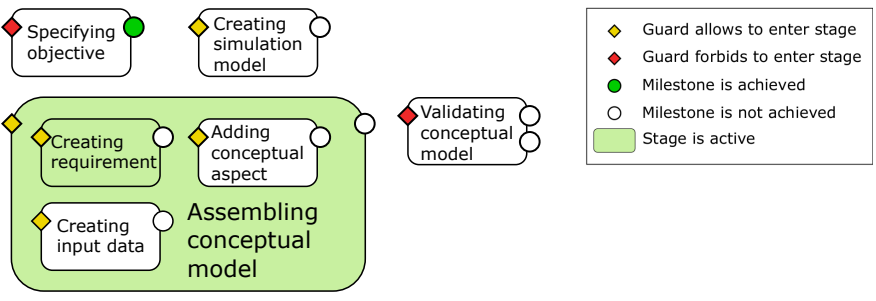

Fig. 7. The Conceptual Model artifact of the artifact-based workflow with all its stages, guards and milestones, adapted from [73]. Since the milestone of the Specifying objective-stage has been achieved, two other stages can be entered: the modeler can start with creating the simulation model or the conceptual model can be (further) assembled. In this example, the modeler enters the Assembling conceptual model-stage and adds a new behavioral requirement As long as the conceptual model is not fully assembled, it cannot be validated and therefore the guard of that stage is disabled.

various artifacts can be used in the automatic generation of simulation experiments.

For instance, in [73] a finite element simulation study of an electrical stimulation chamber was conducted with assistance from the workflow. The collected information could be used to automatically generate a convergence test for this model. The convergence of numerical methods is of high importance in order to retrieve meaningful results from a numerical simulation [74]. In a convergence experiment, the mesh is incrementally refined until the estimated discretization error lies below a given error threshold or until the maximum number of iterations is reached. A meta model for convergence experiments, therefore, requires the following inputs: 1) the region of interest, 2) an error metric allowing to estimate the discretization error for a given region, 3 ) the maximum number of iterations or an error threshold to control the error, 4) an initial meshing hypothesis, i.e., the minimal and maximum size of the finite elements, to initialize the meshing algorithm.

By connecting our pipeline to the workflow system, some of these inputs required by the meta model can be filled automatically by extracting meta-information from the artifacts, e.g., the region of interest and the error metric could have been specified as requirements in the conceptual model [4]. Other inputs are specific to convergence studies, e.g., the initial minimal and maximum size of the elements, and thus need to be filled manually (using our GUI) or with default values (specified in the experiment meta model).

Fig. 8 shows the results of the convergence experiment that was generated semi-automatically for the model of the electrical stimulation chamber. It shows how the observed variable (current at electrode 1) converges with increasing degrees of freedom in the finite element model, and that the refinement could terminate after the fourth iteration.

\section{CONCLusions}

In this paper, we have presented an MDE framework for supporting the conduction of simulation experiments. Central features of the approach are the composition of different types of meta models, a meta model repository, bidirectional code transformations, a variety of tool bindings, and an API.

We have demonstrated how the framework contributes to improving knowledge sharing within but also across the

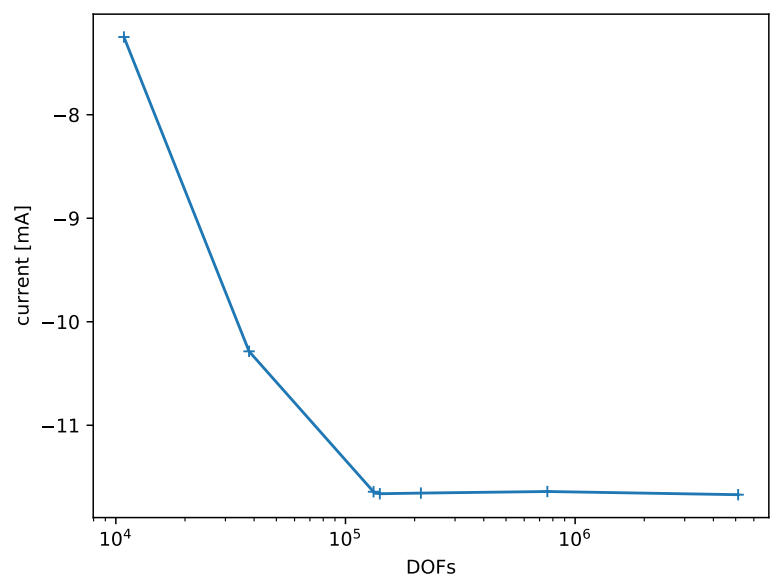

Fig. 8. Convergence of the current w.r.t. the number of degrees of freedom (DOFs). Note that for a large number of DOFs, numerical issues may arise due to the small size of individual elements.

different simulation domains and approaches (i.e., stochastic discrete event simulation of a cell signaling pathway, virtual prototyping of a neurostimulator, and finite element analysis of electric fields). There, the introduction of meta models for base experiments and meta models for complex experiment types proved crucial. Their on-demand composition guided the modeler in specifying complex experiments while catering to rather diverse demands of the respective simulation studies, and thus furthers the productivity of the modeler and the quality of complex experiments. Furthermore, we have shown the framework's practicality for automatically reusing simulation experiments for cross-validation of related models, and for automatically generating and executing simulation experiments, e.g., for conducting convergence tests initiated by a workflow system. We showed that the presented approach fulfills typical expectations associated with model-driven engineering and will be an asset for more effective and systematic simulation studies.

For future work, we plan to increase support for the development of new meta models of base experiments and meta models of complex experiment types, i.e., through the composition and reuse of meta model parts with a clear notion of inheritance. What should become part of a meta model, needs to be debated in the various modeling and simulation communities, possibly via standardization bodies such as SISO [75]. Regarding the automatic support, we plan to build further support components on top of the MDE pipeline, such as an automatic selection and parametrization of experiment types and methods.

\section{ACKNOWLEDGMENT}

The authors would like to thank Fiete Haack for his excellent support in conducting the Wnt experiments.

\section{REFERENCES}

[1] E. Winsberg, Science in the age of computer simulation. University of Chicago Press, 2010. 
[2] O. Balci, "A life cycle for modeling and simulation," Simulation, vol. 88, no. 7, pp. 870-883, Feb. 2012.

[3] R. G. Sargent, "Verification and validation of simulation models," $J$. Simul., vol. 7, no. 1, pp. 12-24, Feb. 2013.

[4] A. Ruscheinski, T. Warnke, and A. M. Uhrmacher, "Artifact-based workflows for supporting simulation studies," IEEE Trans. Knowl. Data Eng., vol. 32, no. 6, pp. 1064-1078, 2020.

[5] B. P. Zeigler, Multifacetted Modelling and Discrete Event Simulation. Academic Press Professional, Inc., 1984.

[6] S. J. E. Taylor, T. Eldabi, T. Monks, M. Rabe, and A. M. Uhrmacher, "Crisis, what crisis - does reproducibility in modeling simulation really matter?" in Proceedings of the 2018 Winter Simulation Conference (WSC), 2018, pp. 749-762.

[7] R. Ewald and A. M. Uhrmacher, "SESSL: A domain-specific language for simulation experiments," ACM Trans. Model. Comput. Simul., vol. 24, no. 2, pp. 1-25, Feb. 2014.

[8] T. Warnke, T. Helms, and A. M. Uhrmacher, "Reproducible and flexible simulation experiments with ML-Rules and SESSL," Bioinformatics, vol. 34, no. 8, pp. 1424-1427, Apr. 2018.

[9] D. Waltemath, R. Adams, F. T. Bergmann, M. Hucka, F. Kolpakov, A. K Miller et al., "Reproducible computational biology experiments with SED-ML - the simulation experiment description markup language," BMC Syst. Biol., vol. 5, no. 1, pp. 1-10, Dec. 2011.

[10] A. Erdemir, T. M. Guess, J. Halloran, S. C. Tadepalli, and T. M. Morrison, "Considerations for reporting finite element analysis studies in biomechanics," Journal of Biomechanics, vol. 45, no. 4, pp. 625-633, 2012.

[11] L. F. Perrone, C. S. Main, and B. C. Ward, "SAFE: Simulation automation framework for experiments," in Proceedings of the 2012 Winter Simulation Conference (WSC), 2012, pp. 1-12.

[12] J. Himmelspach, R. Ewald, and A. M. Uhrmacher, "A flexible and scalable experimentation layer," in Proceedings of the 40th Conference on Winter Simulation (WSC), 2008, pp. 827-835.

[13] J. Salecker, M. Sciaini, K. M. Meyer, and K. Wiegand, "The NLRX R package: A next-generation framework for reproducible netlogo model analyses," Methods in Ecology and Evolution, vol. 10, no. 11, pp. 18541863,2019

[14] A. Teran-Somohano, A. E. Smith, J. Ledet, L. Yilmaz, and H. Oğuztüzün, "A model-driven engineering approach to simulation experiment design and execution," in Proceedings of the 2015 Winter Simulation Conference (WSC), 2015, pp. 2632-2643.

[15] L. Yilmaz, S. Chakladar, and K. Doud, "The goal-hypothesis-experimen framework: A generative cognitive domain architecture for simulation experiment management," in 2016 Winter Simulation Conference (WSC), 2016, pp. 1001-1012.

[16] P. Mohagheghi and V. Dehlen, "Where is the proof? - a review of experiences from applying mde in industry," in Model Driven Architecture Foundations and Applications, I. Schieferdecker and A. Hartman, Eds. Berlin, Heidelberg: Springer Berlin Heidelberg, 2008, pp. 432-443.

[17] R. J. Rodríguez, S. Bernardi, and A. Zimmermann, "An evaluation framework for comparative analysis of generalized stochastic petri net simulation techniques," IEEE Transactions on Systems, Man, and Cybernetics: Systems, vol. 50, no. 8, pp. 2834-2844, 2020.

[18] P. Palensky, E. Widl, and A. Elsheikh, "Simulating cyber-physical energy systems: Challenges, tools and methods," IEEE Transactions on Systems, Man, and Cybernetics: Systems, vol. 44, no. 3, pp. 318-326, 2014.

[19] S. Robinson, "Conceptual modelling for simulation part I: definition and requirements," J. Oper. Res. Soc., vol. 59, no. 3, pp. 278-290, 2008.

[20] P. Wilsdorf, F. Haack, K. Budde, A. Ruscheinski, and A. M. Uhrmacher, "Conducting systematic, partly automated simulation studies - unde venis et quo vadis," in 17th International Conference of Numerical Analysis and Applied Mathematics, 2019.

[21] A. Ruscheinski, K. Budde, T. Warnke, P. Wilsdorf, B. C. Hiller, M. Dombrowsky, and A. M. Uhrmacher, "Generating simulation experiments based on model documentations and templates," in Proceedings of the 2018 Winter Simulation Conference (WSC), 2018, pp. 715-726.

[22] P. Wilsdorf, A. Wolpers, J. Hilton, F. Haack, and A. M. Uhrmacher, "Automatic reuse, adaption, and execution of simulation experiments via provenance patterns," 2021, arXiv:2109.06776 [CS.CE]

[23] J. Bezivin and O. Gerbe, "Towards a precise definition of the omg/mda framework," in Proceedings 16th Annual International Conference on Automated Software Engineering (ASE 2001), 2001, pp. 273-280.

[24] D. Cetinkaya and A. Verbraeck, "Metamodeling and model transformations in modeling and simulation," in Proceedings of the 2011 Winter Simulation Conference (WSC), 2011, pp. 3043-3053.
[25] G. Guizzardi and G. Wagner, "Conceptual simulation modeling with onto-uml advanced tutorial," in Proceedings of the 2012 Winter Simulation Conference (WSC), 2012, pp. 1-15.

[26] G. Kapos, A. Tsadimas, C. Kotronis, V. Dalakas, M. Nikolaidou, and D. Anagnostopoulos, "A declarative approach for transforming sysml models to executable simulation models," IEEE Transactions on Systems, Man, and Cybernetics: Systems, pp. 1-16, 2019.

[27] P. Bocciarelli, A. D' Ambrogio, A. Falcone, A. Garro, and A. Giglio, "A model-driven approach to enable the simulation of complex systems on distributed architectures," SIMULATION, vol. 95, no. 12, pp. 1185-1211, 2019.

[28] H. Vangheluwe and J. de Lara, "Meta-models are models too," in Proceedings of the Winter Simulation Conference, vol. 1, 2002, pp. 597605 vol.1.

[29] O. Dayıbaş, H. Oğuztüzün, and L. Yılmaz, "On the use of model-driven engineering principles for the management of simulation experiments," Journal of Simulation, vol. 13, no. 2, pp. 83-95, 2019.

[30] F. Lorig, Hypothesis-Driven Simulation Studies. Springer Vieweg, Wiesbaden, 2019.

[31] D. Peng, T. Warnke, F. Haack, and A. M. Uhrmacher, "Reusing simulation experiment specifications in developing models by successive composition - a case study of the wnt $/ \beta$-catenin signaling pathway," Simulation, vol. 93, no. 8, pp. 659-677, Apr. 2017.

[32] — "Reusing simulation experiment specifications to support developing models by successive extension," Simulation Modelling Practice and Theory, vol. 68, pp. 33-53, 2016.

[33] J. Cooper, M. Scharm, and G. R. Mirams, "The cardiac electrophysiology web lab," Biophys. J., vol. 110, no. 2, pp. 292-300, Jan. 2016.

[34] P. Wilsdorf, J. Zimmermann, M. Dombrowsky, U. v. Rienen, and A. M. Uhrmacher, "Simulation experiment schemas - beyond tools and simulation approaches," in Proceedings of the 2019 Winter Simulation Conference (WSC), 2019, pp. 2783-2794.

[35] P. A. Fishwick and J. A. Miller, "Ontologies for modeling and simulation: issues and approaches," in Proceedings of the 2004 Winter Simulation Conference (WSC), 2004, p. 264.

[36] S. J. Taylor, D. Bell, N. Mustafee, S. de Cesare, M. Lycett, and P. A. Fishwick, "Semantic web services for simulation component reuse and interoperability: An ontology approach," in Organizational advancements through enterprise information systems: Emerging applications and developments. IGI Global, 2010.

[37] G. A. Silver, J. A. Miller, M. Hybinette, G. Baramidze, and W. S. York, "Demo: An ontology for discrete-event modeling and simulation," SIMULATION, vol. 87, no. 9, pp. 747-773, 2011, pMID: 22919114.

[38] H. Cheong and A. Butscher, "Physics-based simulation ontology: an ontology to support modelling and reuse of data for physics-based simulation," Journal of Engineering Design, vol. 30, no. 10-12, pp. 655687, 2019.

[39] J. Whittle, J. Hutchinson, and M. Rouncefield, "The state of practice in model-driven engineering," IEEE Software, vol. 31, no. 3, pp. 79-85, 2014.

[40] "Unified modeling language," 2020. [Online]. Available: https: //www.omg.org/spec/UML/

[41] D. C. Fallside and P. Walmsley, "XML schema part 0: primer second edition," W3C recommendation, vol. 16, 2004.

[42] "JSON schema specification," 2019. [Online]. Available: https: //json-schema.org/draft/2019-09/release-notes.html

[43] “Object constraint language," 2020. [Online]. Available: https://www. omg.org/spec/OCL/About-OCL/

[44] D. Waltemath, R. Adams, D. A. Beard, F. T. Bergmann, U. S. Bhalla, R. Britten et al., "Minimum information about a simulation experiment (MIASE)," PLOS Comput. Biol., vol. 7, no. 4, pp. 1-4, Apr. 2011.

[45] "Introduction to JSON," 2020. [Online]. Available: http://www.json.org/

[46] "JSON schema validator," 2020. [Online]. Available: https://github.com/ everit-org/json-schema

[47] "Apache FreeMarker manual for Freemarker 2.3.30." 2020. [Online]. Available: https://freemarker.apache.org/docs/index.html

[48] A. M. Law, W. D. Kelton, and W. D. Kelton, Simulation modeling and analysis. McGraw-Hill New York, 2000, vol. 3.

[49] J. Banks, J. S. Carson, B. L. Nelson, and D. M. Nicol, Discrete-Event System Simulation, 5th ed. Prentice Hall, 2010.

[50] T. Székely Jr and K. Burrage, "Stochastic simulation in systems biology," Computational and Structural Biotechnology Journal, vol. 12, no. 20-21, pp. 14-25, 2014.

[51] A. Vachoux, C. Grimm, and K. Einwich, "Analog and mixed signal modelling with systemc-ams," in Proceedings of the 2003 International Symposium on Circuits and Systems (ISCAS), vol. 3, 2003, p. III. 
[52] A. Logg, K.-A. Mardall, and G. N. Wells, Automated Solution of Differential Equations by the Finite Element Method. Springer Science \& Business Media, 2012.

[53] R. E. Caflisch et al., "Monte carlo and quasi-monte carlo methods," Acta numerica, vol. 1998, pp. 1-49, 1998

[54] N. A. Butler, "Optimal and orthogonal Latin hypercube designs for computer experiments," Biometrika, vol. 88, no. 3, pp. 847-857, 10 2001.

[55] T. Crestaux, O. Le Martre, and J.-M. Martinez, "Polynomial chaos expansion for sensitivity analysis," Reliability Engineering \& System Safety, vol. 94, no. 7, pp. 1161-1172, 2009.

[56] T. Homma and A. Saltelli, "Importance measures in global sensitivity analysis of nonlinear models," Reliability Engineering \& System Safety, vol. 52, no. 1, pp. 1-17, 1996.

[57] I. Sobol, "Global sensitivity indices for nonlinear mathematical models and their monte carlo estimates," Mathematics and Computers in Simulation, vol. 55, no. 1, pp. 271-280, 2001, the Second IMACS Seminar on Monte Carlo Methods.

[58] R. Nusse, "Wnt signaling and stem cell control," Cell Research, vol. 18, no. 5, pp. 523-527, 2008 .

[59] H. Clevers and R. Nusse, "Wnt/ $\beta$-catenin signaling and disease," Cell, vol. 149, no. 6, pp. 1192-1205, Jun 2012.

[60] B.-J. Lin, S.-H. Tsao, A. Chen, S.-K. Hu, L. Chao, and P.-H. G. Chao, "Lipid rafts sense and direct electric field-induced migration," Proc. Natl. Acad. Sci. U.S.A., vol. 114, no. 32, pp. 8568-8573, 2017.

[61] F. Haack, H. Lemcke, R. Ewald, T. Rharass, and A. M. Uhrmacher, "Spatio-temporal model of endogenous ros and raft-dependent wnt/betacatenin signaling driving cell fate commitment in human neural progenitor cells," PLOS Comput. Biol., vol. 11, no. 3, pp. 1-28, Mar. 2015.

[62] F. Haack, K. Budde, and A. M. Uhrmacher, "Exploring mechanistic and temporal regulation of LRP6 endocytosis in canonical Wnt signaling," Journal of Cell Science, 2020.

[63] T. Helms, T. Warnke, C. Maus, and A. M. Uhrmacher, "Semantics and efficient simulation algorithms of an expressive multilevel modeling language," ACM Trans. Model. Comput. Simul., vol. 27, no. 2, May 2017.

[64] J. Heller, N. Christoph, F. Plocksties, C. Haubelt, and D. Timmermann, "Towards virtual prototyping of electrically active implants using systemc-ams," in Workshop Methods and Description Languages for Modelling and Verification of Circuits and Systems (MBMV), 2020, p. 29-36.

[65] S. Tennøe, G. Halnes, and G. T. Einevoll, "Uncertainpy: A python toolbox for uncertainty quantification and sensitivity analysis in computational neuroscience," Front. Neuroinform., vol. 12, p. 49, Aug. 2018.

[66] M. Griffin, S. A. Iqbal, A. Sebastian, J. Colthurst, and A. Bayat, "Degenerate wave and capacitive coupling increase human MSC invasion and proliferation while reducing cytotoxicity in an in vitro wound healing model," PLoS One, vol. 6, no. 8, 2011.

[67] K. Budde, J. Zimmermann, E. Neuhaus, M. Schröder, A. M. Uhrmacher, and U. van Rienen, "Requirements for documenting electrical cell stimulation experiments for replicability and numerical modeling," in 201941 st Annual International Conference of the IEEE Engineering in Medicine and Biology Society (EMBC), 2019, pp. 1082-1088.

[68] J. Zimmermann, "EMStimTools," 2020. [Online]. Available: https: //github.com/j-zimmermann/EMStimTools/

[69] E. Lee, A. Salic, R. Krüger, R. Heinrich, and M. W. Kirschner, "The roles of apc and axin derived from experimental and theoretical analysis of the wnt pathway," PLOS Biology, vol. 1, no. 1, p. null, 102003.

[70] M. Hucka, A. Finney, H. M. Sauro, H. Bolouri, J. C. Doyle, H. Kitano, A. P. Arkin, B. J. Bornstein, D. Bray, A. Cornish-Bowden, A. A. Cuellar, S. Dronov et al., "The systems biology markup language (SBML): a medium for representation and exchange of biochemical network models," Bioinformatics, vol. 19, no. 4, pp. 524-531, 032003.

[71] R. S. Malik-Sheriff, M. Glont, T. V. N. Nguyen, K. Tiwari, M. G Roberts, A. Xavier, M. T. Vu, J. Men, M. Maire, S. Kananathan, E. L. Fairbanks, J. P. Meyer et al., "BioModels - 15 years of sharing computational models in life science," Nucleic Acids Research, vol. 48, no. D1, pp. D407-D415, 2020

[72] T. U. Consortium, "UniProt: a worldwide hub of protein knowledge," Nucleic Acids Research, vol. 47, no. D1, pp. D506-D515, 112018

[73] A. Ruscheinski, P. Wilsdorf, J. Zimmermann, U. van Rienen, and A. M. Uhrmacher, "An artifact-based workflow for finite-element simulation studies," 2020, arXiv:2010.07625 [CS.CE].

[74] P. Morin, R. H. Nochetto, and K. G. Siebert, "Convergence of adaptive finite element methods," SIAM Review, vol. 44, no. 4, pp. 631-658, 2002.
[75] "Simulation interoperability standards organization." [Online]. Available: https://www.sisostds.org/

Pia Wilsdorf received a M.Sc. degree in Computer Science from the University of Rostock in 2018. She is currently pursuing a Ph.D. degree in the Modeling and Simulation group at the University of Rostock. Her research focuses on automatic support for conducting simulation studies using knowledge-centered approaches.

Jakob Heller received his M.Sc. degree in Electrical Engineering in 2018 Currently, he works as a doctoral researcher for the CRC 1270 ELAINE at the Institute of Applied Microelectronics and Computer Engineering at the University of Rostock. The Focus of his research is on chronic evaluation of neural field potentials and neurostimulators.

Kai Budde studied Interdisciplinary Sciences at the Swiss Federal Institute of Technology (ETH) Zurich and Physics at the University of Rostock and received his M.Sc. degree in Physics from the University of Rostock. $\mathrm{He}$ is currently a Ph.D. student in the Modeling and Simulation group at the University of Rostock working within the CRC 1270 ELAINE. His research focuses both on in silico and on experimental in vitro methods for understanding the effects of electrical stimulation on cells.

Julius Zimmermann studied Physics at the University of Rostock and received his master's degree in 2017. Since then, he has been pursuing a $\mathrm{Ph} . \mathrm{D}$. degree in Electrical Engineering at the same university. His research focuses on numerical simulations of electrical stimulation, particularly with application in cartilage tissue engineering.

Tom Warnke received his M.Sc. degree and his Dr.-Ing. degree in Computer Science from the University of Rostock. He works as a Postdoctoral researcher in Adelinde M. Uhrmacher's Modeling and Simulation group. His research interests include the design and implementation of domain-specific languages for specifying and conducting simulation experiments.

Christian Haubelt received his diploma degree in Electrical Engineering from the University of Paderborn, Germany, in 2001. He finished his Dr.-Ing. in Computer Science and his Habilitation (postdoctoral lecture qualification) in Computer Engineering at University of Erlangen-Nuremberg, Germany, in 2005 and 2010, respectively. Since 2011, he is a Professor of Embedded Systems at the University of Rostock, Germany. His research interests include embedded and cyber-physical system design, virtual prototyping, smart sensor systems, and multi-objective optimization.

Dirk Timmermann studied Electrical Engineering at the University of Dortmund, Germany. In 1990 he received his Dr.-Ing. degree from the Department of Electrical Engineering at the University of Duisburg, Germany. Since 1994 he is a University Professor in Computer Engineering at the University of Rostock, Germany, and director of the Institute of Applied Microelectronics and Computer Engineering. His research focuses on energy aware digital CMOS circuits and systems, wireless sensor networks, and embedded middleware architectures.

Ursula van Rienen (M'01) received the venia legendi for the fields "Electromagnetic Field Theory" and "Scientific Computing" at the Technische Universität Darmstadt. Since 1997, she holds the chair in "Electromagnetic Field Theory" at the University of Rostock. Her research work is focused on computational electromagnetics with various applications, ranging from biomedical engineering to accelerator physics.

Adelinde M. Uhrmacher is professor at the Institute for Visual and Analytic Computing at the University of Rostock and head of the modeling and simulation group since 2000. She has been on the editorial board of diverse simulation-related journals and conferences, e.g., editor in chief of SCS Simulation (2000-2006), and editor in chief of the ACM Transactions on Modeling and Computer Simulation since 2013. Her research focuses on the development of methods for multi-level modeling, simulation, and conducting simulation studies, and their application in areas such as cell biology and demography. 